-Editorial·

\title{
Neurodevelopment and degeneration
}

\author{
Margaret S. $\mathrm{Ho}^{*}$ \\ Department of Anatomy and Neurobiology, School of Medicine, Tongji University, Shanghai 200092, China \\ *Guest Editor of the Special Issue, Associate Editor. E-mail: margaret_ho@tongji.edu.cn \\ (C) Shanghai Institutes for Biological Sciences, CAS and Springer-Verlag Berlin Heidelberg 2014
}

Over the past decades, continuous effort has been made to resolve unsettled matters in the emerging scene of neuronal development. Despite the rapid progress, neuroscientists remain fascinated by the intricate networks of signaling pathways and molecules conserved among species. Fundamental questions such as progenitor cell specification, compartmentalized cellular tactics, and the identification of factors involved in orchestrating the progression of development are being actively pursued. Furthermore, reversing the effects of development, neurodegeneration symbolizes an opposite force in limiting growth and has been linked to a variety of diseases. To date, a tremendous amount of work from the research community has conceptualized the central image of how nervous systems develop and are equipped to modulate animal functions, albeit with mysterious gaps in the picture that remain to be filled.

In this issue, we have compiled a set of articles that cover topics ranging from neural stem cell (NSC) specification and differential axon/dendrite growth, to signaling molecules that participate in neuronal morphogenesis, synaptic refinement, and behavioral function. Our issue aims to discuss recent progress in the fundamental principles regulating neurodevelopment and degeneration, with the exploitation of model organisms such as Drosophila and Caenorhabditis elegans. Below, I highlight these contributions in subcategories, to provide our readers with a comprehensive overview.

\section{Basic Unit Assembly}

As the recognized building block of the nervous system, the neuron is compartmentalized into axons and dendrites that mediate information processing. Another major cell type, glia, though they do not exhibit major polarized features, play significant roles in regulating neuronal development and function. The origin of these cells and how they are generated from NSCs have long been an active area of research. Song et al. describe the development of adult hippocampal NSCs and their progeny ${ }^{[1]}$. These NSCs exhibit distinct features during different developmental stages. Understanding the mechanism of their specification, development, and regulation provides cues to their therapeutic potential in regard to neuronal regeneration and degeneration. In addition, activity-dependent mechanisms and the required elements from the niche microenvironment for regulating the development of these NSCs are illustrated $^{[1]}$. Regulatory factors such as neurotransmitters, morphogens, and transcription factors are thoroughly discussed $^{[1]}$.

Once specified and polarized, neuronal compartments such as axons and dendrites begin to undergo differentiallyregulated growth. Ye et al., using both mammals and flies as models, discuss the underlying mechanisms regulating axon and dendrite growth ${ }^{[2]}$. Two models, dedicated and bimodal mechanisms, have been proposed and are reviewed. Whereas the dedicated regulators like BMP7 or Rac1 affect only the growth of either axons or dendrites, bimodal regulators like Sema3A execute binary functions that promote axon or dendrite growth while inhibiting the other $^{[2]}$. Accompanying growth, differentiated axons exhibit guidance properties that Liu et al. review in great detail ${ }^{[3]}$. A subject of interest for many years, axon guidance is crucial for neuronal function and the microtubule dynamics at the growth cone of axons has been considered as a classical paradigm for understanding cell motility and dynamics ${ }^{[3]}$.

In addition to neurons, a plethora of evidence has suggested that glia play pivotal roles during 
neurodevelopment, synaptic function, and plasticity. Ho et al. explore glial functions in Drosophila, summarizing recent research advances in this particular field ${ }^{[4]}$.

\section{At the Synapse}

The proper organization and assembly of a functional synapse is important for neuronal function throughout developmental stages. Wang et al. provide experimental evidence that surface-located GluN2A-containing NMDARs, but not those containing GluN2B, cluster at the synaptic site and the clustering is mediated by the carboxyl-terminus ${ }^{[5]}$. Furthermore, GluN2A-containing NMDARs preferentially associate with PSD-95, suggesting a pivotal role for the synaptic localization of NMDARs during neuronal development and function ${ }^{[5]}$.

Throughout development, synapses undergo dynamic changes including growth-opposing actions like elimination in order to become truly functional. Caspases, initially recognized for their apoptotic roles during neurodegeneration, have emerged as important regulators of synaptic refinement and elimination. In the Perspective, Luo et al. discuss the new theme regarding the functions of caspase- 3 in mammalian neuromuscular junction and the central nervous system ${ }^{[6]}$. In both cases, caspase- 3 participates in synaptic refinement via controlling the $\mathrm{ACh}$ cluster dispersion or AMPA receptor internalization, leading to spine elimination ${ }^{[6]}$.

Drug-induced animal behavior has been frequently used to investigate the mechanism that directly links to a synaptic component, providing further implications on how a synapse is developed and structured for function. Li et al. thoroughly review the utility of the animal model C. elegans to study the underlying mechanism of alcohol addiction $^{[7]}$. A nice summary of synaptic factors such as BK channels, receptors and neurotransmitters, and the lipid microenvironment is provided in this review and the correlation between drug-induced behavior and synaptic development is also discussed ${ }^{[7]}$.

\section{Epigenetic and Transcriptional Regulation}

Identifying the factors involved in neurodevelopment and neurodegeneration has been a continuous task in the field. Cheng et al. summarize the function of one such factor, methyl-CpG-binding protein 2 (MeCP2), during neurodevelopment ${ }^{[8]}$. A classic methylated-DNA binding protein, MeCP2 represses transcription, participates in nuclear microRNA processing, and has been implicated in various neurodevelopmental disorders such as Rett syndrome and autism spectrum disorder ${ }^{[8]}$. Posttranslational modifications of MeCP2 itself have also provided extra layers of complexity in regulating MeCP2 function during neurodevelopment ${ }^{[8]}$.

Doxakis then discusses a set of RNA-binding proteins (RBPs) essential for brain development and function ${ }^{[9]}$. RBPs participate in the mechanism of pre-mRNA splicing to produce diversity, local mRNA translation to provide control over protein expression, and fine-tuning mRNA translation by alternative polyadenylation ${ }^{[9]}$. Intriguingly, RBPs have been implicated in a number of neurodegenerative diseases, based upon studies of their localization patterns and mutagenic analysis.

\section{Neurodegeneration: Action and Response}

Nervous systems evolve self-defense mechanisms to protect the integrity of the cellular environment to act and function properly. To face dangerous situations such as neurodegeneration, explicit control over networks of proteins is required for strategic planning and problemsolving. Sealing of the axolemmal membrane upon mechanical trauma serves as one good example. Shi et al. provide a thorough review on the membrane-sealing mechanisms upon injury, providing insights into how neuronal membranes react to injury and self-heal ${ }^{[10]}$. In this article, two types of models of membrane integrity are discussed: the line tension and the membrane tension mechanisms. In a different scenario, Hsueh et al. discuss how neurons execute the innate immunity program mediated by the Toll-like receptors and their adaptor proteins like Sarm1 upon foreign pathogenic attack ${ }^{[11]}$. These signaling molecules regulate neuronal morphology and function in the absence of an immune challenge, and also play significant roles during neurodegeneration.

This special issue on "Neurodevelopment and Degeneration" presents a collection of articles that cover the signaling mechanisms for cell specification, differential growth, and information delivery via synaptic organization. It is not surprising that these mechanisms also contribute to 
disease or injury states like neurodegeneration. We hope that the topics covered here will bring readers insights on the fundamental principles underlying neurodevelopment, and at the same time ignite the passion of our fellow scientists to address the intriguing questions that remain mysterious in the field.

\section{REFERENCES}

[1] Crowther AJ, Song J. Activity-dependent signaling mechanisms regulating adult hippocampal neural stem cells and their progeny. Neurosci Bull 2014, 30: 542-556.

[2] Wang X, Sterne GR, Ye B. Regulatory mechanisms underlying the differential growth of dendrites and axons. Neurosci Bull 2014, 30: 557-568.

[3] Liu G, Dwyer T. Microtubule dynamics in axon guidance. Neurosci Bull 2014, 30: 569-583.

[4] Ou J, He Y, Xiao X, Yu TM, Chen C, Gao Z, et al. Glial cells in neuronal development: recent advances and insights from Drosophila melanogaster. Neurosci Bull 2014, 30: 584-594.

[5] Yan YG, Zhang J, Xu SJ, Luo JH, Qiu S, Wang W. Clustering of surface NMDA receptors is mainly mediated by the C-terminus of GluN2A in cultured rat hippocampal neurons. Neurosci Bull 2014, 30: 655-666.

[6] Wang JY, Luo ZG. Non-apoptotic role of caspase-3 in synapse refinement. Neurosci Bull 2014, 30: 667-670.

[7] Zhu G, Zhang F, Li W. Nematodes feel a craving - Using Caenorhabditis elegans as a model to study alcohol addiction. Neurosci Bull 2014, 30: 595-600.

[8] Cheng TL, Qiu Z. MeCP2: multifaceted roles in gene regulation and neural development. Neurosci Bull 2014, 30: 601-609.

[9] Doxakis E. RNA binding proteins: a common denominator of neuronal function and dysfunction. Neurosci Bull 2014, 30: 610-626.

[10] Hendricks BK, Shi R. Mechanisms of neuronal membrane sealing following mechanical trauma. Neurosci Bull 2014, 30: 627-644.

[11] Liu HY, Chen CY, Hsueh YP. Innate immune responses regulate morphogenesis and degeneration: roles of Toll-like receptors and Sarm1 in neurons. Neurosci Bull 2014, 30: 645-654. 